\title{
Morusin induces osteogenic differentiation of bone marrow mesenchymal stem cells by canonical Wnt/ $\beta$-catenin pathway and prevents bone loss in an ovariectomized rat model
}

Ming Chen ${ }^{1,2+}$, Hui Han ${ }^{1,2+}$, Siqi Zhou ${ }^{1,2,3}$, Yinxian Wen ${ }^{1,2^{*}}$ and Liaobin Chen ${ }^{1,2^{*}}$

\begin{abstract}
Background: Osteoporosis (OP) is a metabolic bone disease due to the imbalance of osteogenesis and bone resorption, in which, bone marrow mesenchymal stem cells (BMSCs) have a significant effect as the seed cells. Recent research has shown the function of Morusin on inhibiting osteoclast differentiation in vitro. However, whether Morusin can regulate the osteogenic differentiation in addition to the proliferation of BMSCs remains unclear.
\end{abstract}

Methods: BMSCs were isolated from 4-week-old Wistar rats and then treated with different concentrations of Morusin for 3, 5, 7, and 14 days. The proliferation of BMSCs was detected by MTT assay. The effect of Morusin on osteogenic differentiation of BMSCs was detected by RT-qPCR, Western blotting, ALP, and Alizarin Red staining. The effect of Morusin on Wnt/ $\beta$-catenin signaling pathway was analyzed by RT-qPCR, Western blotting, and immunofluorescence. Finally, in the ovariectomy-induced osteoporosis model, the anti-osteoporosis activity of Morusin was determined by micro- $\mathrm{CT}, \mathrm{HE}$, and immunohistochemistry.

Results: The results showed the function of 2.5-10 $\mu \mathrm{M}$ Morusin in the promotion of the proliferation in addition to osteogenic differentiation of BMSCs. Moreover, it also has an impact in activating the Wnt/ß-catenin signaling pathway via inhibition of $\beta$-catenin phosphorylation as well as promotion of its nuclear translocation. Upon Dickkopf-related protein-1 (DKK-1, an inhibitor of the Wnt/B-catenin signaling pathway) was added to the Morusin, Morusin had a decreased stimulatory osteogenic effect on BMSCs. Finally, in the rat OP model, we found that Morusin could also exert anti-osteoporosis activity in vivo.

Conclusions: This study indicates the ability of Morusin in the promotion of osteogenic differentiation of BMSCs via the activation of Wnt/ $\beta$-catenin signaling pathway and also shows the potential of Morusin to be an agent for osteoporosis treatment.

Keywords: Morusin, BMSCs, Wnt/ß-catenin, Osteoporosis

\footnotetext{
*Correspondence: wenyinxian@whu.edu.cn; Ibchen@whu.edu.cn

${ }^{\dagger}$ Ming Chen and Hui Han contributed equally to this work.

'Department of Joint Surgery and Sports medicine, Zhongnan Hospital of Wuhan University, Wuhan 430071, China

Full list of author information is available at the end of the article
}

(C) The Author(s). 2021 Open Access This article is licensed under a Creative Commons Attribution 4.0 International License, which permits use, sharing, adaptation, distribution and reproduction in any medium or format, as long as you give appropriate credit to the original author(s) and the source, provide a link to the Creative Commons licence, and indicate if changes were made. The images or other third party material in this article are included in the article's Creative Commons licence, unless indicated otherwise in a credit line to the material. If material is not included in the article's Creative Commons licence and your intended use is not permitted by statutory regulation or exceeds the permitted use, you will need to obtain permission directly from the copyright holder. To view a copy of this licence, visit http://creativecommons.org/licenses/by/4.0/ The Creative Commons Public Domain Dedication waiver (http://creativecommons.org/publicdomain/zero/1.0/) applies to the data made available in this article, unless otherwise stated in a credit line to the data. 


\section{Introduction}

Osteoporosis (OP), a highly prevalent metabolic bone disease, is featured with reduced bone mineral content in addition to structural degeneration of the skeletal system $[1,2]$. Generally speaking, OP is a dynamic pathological state resulted from the altered homeostasis between bone resorption of osteoclasts and bone formation of osteoblast [3]. Bone marrow mesenchymal stem cells (BMSCs), considered as pluripotent mesenchymal cells, possess the ability of multilineage differentiation, i.e., the tendency to differentiate into cells of varied categories, such as osteoclasts, chondrocytes, osteoblasts, myocytes, and hepatocytes [4]. To date, there are increasing evidences that the inhibition of osteoblastic activity and differentiation is mainly due to the reduced proliferative potential of BMSCs, especially in the elderly [5-7]. Thus, promoting the osteogenesis of BMSCs may be a promising strategy for OP treatment.

The Wnt/ $\mathrm{W}$-catenin signaling pathway is a significant classical pathway in osteogenic differentiation of BMSCs [8]. As a member of the secretory lipid modification signaling glycoproteins family, Wnt is caused to bind to the receptor mainly by palmitoylation. The secreted Wnts (such as Wnt3a) would activate the typical Wnt/ $\beta$-catenin signaling pathway, when it binds to low density lipoprotein receptor- associated protein 5 (LRP5) or LRP6 and Frizzled transmembrane receptors $[9,10]$. Then, a series of cascade reactions lead to cytosolic instability of $\beta$ catenin, which then migrates to the nuclei and stimulates osteogenic gene transcription. According to the previous reports, the TLR4 deficiency in knockout mice could promote fracture healing and increase bone mass by way of activating the $\mathrm{Wnt} / \beta$-catenin signaling pathway [11]. Another study revealed that vasoactive intestinal peptide (VIP) promoted the BMSCs osteogenesis in addition to the angiogenesis differentiation in vitro through activating the Wnt/ $\beta$-catenin signaling pathway, and advanced bone recovery in vivo [12]. All these evidences suggest $\mathrm{Wnt} / \beta$ catenin signaling pathways are vital for bone mass maintenance, osteogenic differentiation, and promotion of bone repair.

Morusin (Fig. 1a) is one of the main active substances isolated from mulberry bark, which was proved to have antitumor, anti-inflammatory, and antifungal activities [13-15]. Combined with previous researches, we found that Morusin could inhibit the progression of human osteosarcoma by means of the phosphatidylinositol 3'-kinase(PI3K)-Akt signaling pathway [16]. Moreover, its effect on bone metabolism is gradually revealed. A recent study identified a novel method for the synthesis of Morusin and evaluated the negative regulation of Morusin in the osteoclast differentiation in vitro [17]. However, its effect on BMSCs osteogenic differentiation and its possible molecular mechanisms have not been fully illustrated. Herein, we examined the impact and underlying molecular mechanism of Morusin in OP, with the purpose of determining whether Morusin would be a potential candidate for the OP treatment.

\section{Materials and methods}

Chemicals and reagents

We allowed Morusin, purchased from MedChemExpress (MCE, New Jersey, USA), to dissolve in

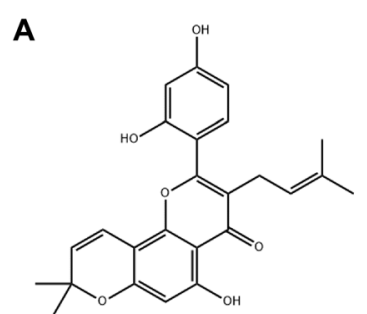

D

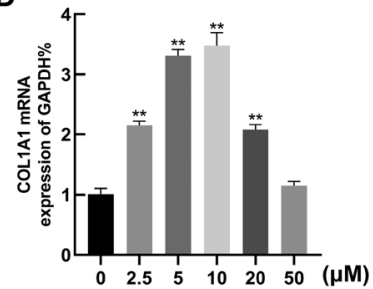

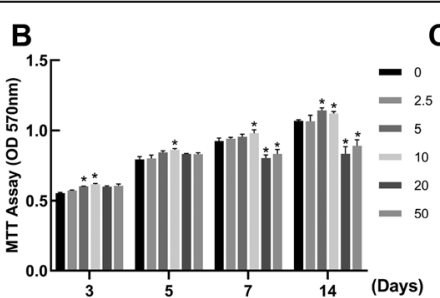

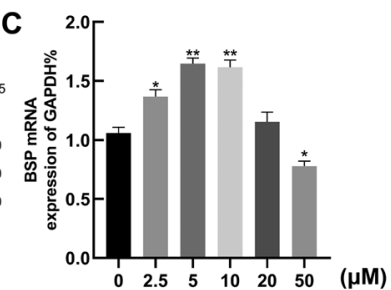

E

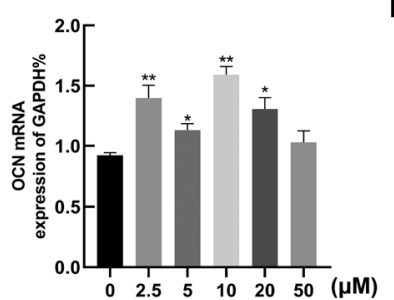

$\mathbf{F}$

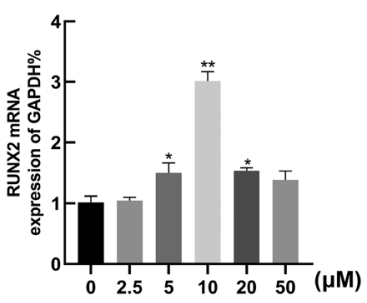

Fig. 1 The impacts of Morusin on proliferation and osteogenic differentiation of BMSCs. a The chemical structure of Morusin. $\mathbf{b}$ Detection of the proliferation toxicity of Morusin on BMSCs via MTT assay. BMSCs were cultured in osteogenic growth medium (OGM) with diverse concentrations of Morusin for 3, 5, 7, and 14 days. c-f mRNA level of marker genes for osteogenesis. Quantitative real-time PCR analysis of gene expression of osteogenic differentiation markers. We incubated BMSCs with Morusin of diverse concentrations $(0-50 \mu \mathrm{M})$ for 3 days to observe the impact of Morusin on osteogenic differentiation. BMSCs, bone marrow mesenchymal stem cells; RUNX2, runt-related transcription factor 2; COL1A1, collagen type I alpha 1; BSP, bone sialoprotein; OCN, osteocalcin. We repeated the independent experiment for three times, with similar results achieved each time. Means $\pm \mathrm{SEM}$. ${ }^{*} P<0.05$ and ${ }^{*} P<0.01$ in comparison to the control group 
dimethylsulfoxide (DMSO) and dilute in cell culture solution to make the DMSO content less than $0.1 \%$ of the total amount. We procured the penicillin/streptomycin, Trizol, and reverse transcription kit from Thermo Fisher Scientific (Waltham, USA). We ordered the $\alpha$-Minimum Essential Medium ( $\alpha$-MEM) and Fetal Bovine Serum (FBS) from HyClone Co. (Logan, USA). Rat Dickkopfrelated protein 1 (DKK1) was obtained from R\&D Systems (Waltham, USA) [18]. The antibody for runtrelated transcription factor 2 (RUNX2, sc-390351) was ordered from Santa Cruz Biotechnology Co. (Dallas, USA). The collagen type I alpha 1(COL1A1, A16891), phosphorylated $\beta$-catenin ( $p$ - $\beta$-catenin, AP0579), and $\beta$ catenin (A19657) antibody was obtained from ABclonal Technology Co., Ltd. (Wuhan, China). The alizarin red staining kit and alkaline phosphatase chromogenic kit was ordered from Beyotime Co., Ltd. (Shanghai, China). The synthesis of all primers was performed by Tianyihuiyuan Biotech Co., Ltd. (Wuhan, China). As to other reagents, we selected the ones of analytical grade for use in experiments.

Isolation, culture, differentiation, and treatment of BMSCs BMSCs were derived from specific pathogen-free (SPF) Wistar rats after isoflurane anesthesia through femoral irrigation in this study. The schemes for isolation, culture, and osteogenic differentiation of BMSCs followed previous researches [19], and the osteogenic growth medium (OGM) included $\alpha$-MEM, $10-20 \%$ FBS, and $100 \mathrm{IU} / \mathrm{ml}$ penicillin/streptomycin. Then, treatment of the cells was conducted with Morusin at diverse concentrations $(0,2.5,5,10,20$, and $50 \mu \mathrm{M})$ in the osteogenic differentiation medium (ODM, $\alpha$-MEM with $0.1 \mu \mathrm{M}$ hexadecadrol, 10-20\% FBS, $50 \mathrm{mMl}$-ascorbic acid-2phosphate, $100 \mathrm{IU} / \mathrm{ml}$ penicillin streptomycin combination, and $10 \mathrm{mM} \beta$-glycerophosphate) to determine the optimal concentration of Morusin for increasing proliferation and osteogenic differentiation of BMSCs. Next, $500 \mathrm{ng} / \mathrm{ml}$ of Dickkopf-related protein-1 (DKK-1, an inhibitor of the $\mathrm{Wnt} / \beta$-catenin signaling pathway) was applied for investigating the involvement of $\mathrm{Wnt} / \beta$-catenin pathway in Morusin promoting the differentiation and proliferation of BMSCs [20].

\section{BMSC proliferation assay}

To observe the impact of diverse Morusin concentrations in BMSC proliferation, cells were inoculated in 96-well plates $\left(1 \times 10^{4}\right.$ per well $)$ and MTT assay was performed. Next, we added Morusin $(0,2.5,5,10,20$, and $50 \mu \mathrm{M})$ to cells in OGM on 3, 5, 7, and 14 days. At each time point, we added $20 \mu \mathrm{L}$ MTT $(5 \mathrm{mg} / \mathrm{mL})$ reagents to each well for incubating the cells at $37^{\circ} \mathrm{C}$ for $2 \mathrm{~h}$. The viability of BMSCs was evaluated by a microplate reader (Thermo Fisher Scientific, USA) through the measurement of the absorbance at $570 \mathrm{~nm}$ after the formazan crystals were dissolved in DMSO.

\section{ALP staining and alizarin red staining(ARS)}

BMSCs were plated on 12-well plates at a density of $1 \times$ $10^{4}$ per well and differentiated by ODM for 3 or 10 days, respectively. After fixing the cells with $4 \%$ paraformaldehyde for $30 \mathrm{~min}$, we used PBS for washing the cells for three times, and then, we adopted alkaline phosphatase chromogenic kit to incubate the cells at room temperature in darkness for $1 \mathrm{~h}$ for staining. Finally, we employed an inverted microscope (Olympus, Japan) for observing and photographing the staining results.

ARS was used to detect mineral deposition after BMSCs induced differentiation. We inoculated the cells on 6-well plates with a density of $1 \times 10^{6}$ per well and differentiated them by ODM for 14 days. Then, the cells were fixed and washed as aforementioned. After that, we added Alizarin Red staining solution to each well of the 6 -well plate for $15 \mathrm{~min}$. They were then rinsed again with PBS and the calcium salt deposition were observed via an inverted microscope (Olympus, Japan).

\section{Total RNA extraction and quantitative real-time PCR (RT- qPCR)}

After osteogenesis induction, we used PBS for washing BMSCs before adding Trizol reagent to isolate total RNA from BMSCs. We determined the purity and concentration of the isolated RNA via a Nano-Drop-2000 nucleic acid analyzer. Then we adopted a reverse transcription kit for conversion of the total RNA into cDNA. We evaluated the expression levels of mRNA by onestep RT-qPCR with thermal Cycler (ABI Step One, USA). Then, we applied the $2^{-\Delta \Delta C t}$ method for analysis of the target genes in terms of the expression level before we normalized all values to the expression level of glyceraldehyde phosphate dehydrogenase (GAPDH) mRNA. Table 1 described the primer sequences of every group of genes applied in this experiment.

\section{Western blot (WB)}

We used precooled PBS for washing the cells for thrice. We, then, adopted $150 \mu \mathrm{l}$ RIPA Lysis Buffer and $1 \mathrm{mM}$ protease inhibitor for lysing the cells for $25 \mathrm{~min}$ on ice to extract the total protein. We separated the same amounts of proteins using $10 \%$ or $12.5 \%$ sodium dodecyl sulfate polyacrylamide gel electrophoresis (SDS-PAGE) before transferring them to polyvinylidene difluoride (PVDF) membranes. 3\% bovine serum albumin (BSA) or $5 \%$ skim milk was employed for blocking the membranes for $60 \mathrm{~min}$. Then, we adopted primary antibodies for membrane incubation at $4{ }^{\circ} \mathrm{C}$ for $16 \mathrm{~h}$, including GAPD $\mathrm{H}$ (1:2000 dilution), RUNX2 (1:300 dilution), $p-\beta$ catenin (1:1000 dilution), $\beta$-catenin (1:1000 dilution), 
Table 1 The primer sequences of genes in this experiment

\begin{tabular}{lll}
\hline Target genes & Forward primer & Reverse primer \\
\hline COL1A1 & TGTCGTTCAACGGCACAG & TGTGGTAGACTCCACGACA \\
RUNX2 & GAGCGTTCAACGGCACAG & GACAGTAGACTCCACGACA \\
BSP & CGTCTCCATGGTGGATATG & GGATGTAGTTCTGCTCATGG \\
OCN & GTCAGACTACAACATCCAGAAG & CGAGTATCTTCCTGTTGACC \\
$\beta-$ Catenin & GCCATCACCACGCTGCATAATCT & GGCAGTCTGTCGTAATAGCCAAGAA \\
DVl-1 & CCTTCCATCCAAATGTTGCAGTA & GGGCAGCCTCATCACGGTT \\
LEF-1 & CACACAACTGGCATCCCTCATC & GCTCCTGTTCCTTCTCTCTGTTCGT \\
GAPDH & GGGTGTGAACCACGAGAAAT & ACTGTGGTCATGAGCCCTTC \\
\hline
\end{tabular}

COL1A1 collagen type I alpha 1, RUNX2 runt-related transcription factor 2, BSP bone sialoprotein, OCN osteocalcin, DVI-1 dishevelled-1, LEF-1 lymphoid enhancing factor-1, GAPDH glyceraldehyde phosphate dehydrogenase

and COL1A1(1:1000 dilution). TBST was applied in membrane washing for 3 times ( 5 min each) and a fluorescence-conjugated secondary antibody (1:5000 dilution) was employed for membrane incubation for $1 \mathrm{~h}$. Finally, enhanced chemofluorescence reagents were used for visualization of immunoreactive protein signals.

\section{Immunofluorescence analysis}

Precooled PBS was used for washing BMSCs cultured in 6-well plates for 3 times. Then, we adopted $4 \%$ paraformaldehyde to fix BMSCs at room temperature for $15 \mathrm{~min}$. After we washed the cells for twice with PBS, we implemented $0.5 \%$ Triton $\mathrm{X}-100$ to permeabilize the cells for $15 \mathrm{~min}$ and employed 3\%BSA to block the cells at room temperature for $40 \mathrm{~min}$. We washed the blocked BMSCs before their overnight incubation at $4{ }^{\circ} \mathrm{C}$ for $12 \mathrm{~h}$ with the primary antibodies of COL1A1(1:200 dilution), RUNX2(1: 50 dilution), and $\beta$-catenin (1:200 dilution) protein. Again, we used PBS for washing the cells for 3 times before their incubation with a fluorescein-linked secondary antibody (1:100 dilution) for $40 \mathrm{~min}$ at room temperature. Then, we stained the nuclei with 4',6-diamidino-2-phenylindole (DAPI, Thermo Fisher Scientific, Waltham, USA) for 5 min. Finally, we observed the cellular samples through a fluorescence microscope (Nikon, Japan).

\section{Ovariectomized (OVX) rat model}

We purchased all specific pathogen-free Wistar rats [female, aged 16 weeks and weighted 230-360 g, NO.20200018, license number: SCXK (Hubei), certification number: 42000600038191] from the Experimental Center of the Hubei Medical Scientific Academy (Wuhan, China). All animal experiments were conducted in the Center for Animal Experiment of Wuhan University (Wuhan, China), and all protocols were authorized by the Animal Welfare Committee in Wuhan University (License number: 14016).

Firstly, we randomly classified 15 female Wistar rats into three groups: Sham operated group, OVX group, and OVX $\mathcal{E}$ Morusin $(40 \mathrm{mg} / \mathrm{kg})$ group [21]. Both the
OVX group and the OVX \& Morusin group received ovariectomies including bilateral ovaries, ovarian capsule, partial fallopian tubes, and fed with a calciumdeficient diet $(0.1 \%$ calcium and $0.77 \%$ phosphorus $)$ to induce experimental osteoporosis, whereas the Sham operated group underwent laparotomy without oophorectomy and fed with a standard diet. At 6 weeks following OVX, Morusin $(40 \mathrm{mg} / \mathrm{kg}$, dissolved by DMSO and then diluted by saline, with a final DMSO concentration of $5 \%)$ intragastric administration was administered to rats in the OVX \& Morusin group every 5 days, while rats in the Sham and OVX group were intragastricly administrated with 5\% dimethylsulfoxide (DMSO) in saline. After 4 weeks of Morusin treatment, the rats were euthanized and sacrificed; then, we collected the femurs. After that, the left side of femurs was soaked in $4 \%$ paraformaldehyde for 2 days at $4{ }^{\circ} \mathrm{C}$ for subsequent pathological testing, while the right was applied for micro-CT and other tests.

\section{Histological and immunohistochemistry analysis}

Femurs were fixed overnight with $4 \%$ paraformaldehyde before being dehydrated and embedded in paraffin. Then, hematoxylin and eosin (HE) was used for femur section staining to observe bone trabecular area and related bone mass indexes [osteoblasts number/bone perimeter (N.Ob/B.Pm) and osteoblast surfaces/bone surface (Ob.S/BS)]. Then, immunohistochemistry was performed to determine the level of RUNX2 protein in the femur. The dilution of RUNX2 primary antibody was 1:100. An optical microscope (Olympus, Japan) was implemented for the staining result observation and photographing. Then, we analyzed the stained images with image J software (version 6.0) before measurement of the mean optical density (MOD) of 5 random fields in every section for determining the staining intensity.

\section{Micro-CT scanning}

Femurs fixed in 75\% alcohol were scanned and analyzed with skyscan1276 $\mu \mathrm{CT}$ system (Bruker, Germany). The sample was placed in a transparent cylindrical groove 
and secured with paper tape. The scanning mode was set to $0.5-\mathrm{mm}$ filter, $450-\mathrm{V}$ scanning voltage, and $5-\mu \mathrm{m}$ scanning resolution. After scanning data reconstruction, the region of interest was selected for non-volumerelated parameter analysis [trabecular separation (Tb.Sp), trabecular number (Tb.N), bone volume/tissue volume (BV/TV), connectivity density (Conn.Dens.), trabecular thickness (Tb.Th), and structure model index (SMI)] and reconstructed to obtain 3D images [22].

\section{Data analysis}

We indicated all experimental values as mean \pm standard error of mean (SEM) of the experimental values (we repeated each experiment for three times). For comparison between the two groups, we performed double-tailed Student $t$ test to determine the statistical significance. For comparison between more than two groups, we implemented one-way ANOVA. A $p<0.05$ showed statistically significant difference.

\section{Results}

The influence of Morusin in the proliferation and osteogenesis differentiation of rat BMSCs in vitro

During BMSC proliferation, Morusin at diverse concentrations $(0-50 \mu \mathrm{M})$ was treated for 3-14 days. Then, we executed MTT assay. As per the results, there was promoted proliferation detected in the osteogenic BMSCs treated with Morusin at 2.5, 5, and $10 \mu \mathrm{M}$ (Fig. 1b) in days $3,5,7$, and 14 , while Morusin of $20 \mu \mathrm{M}$ and $50 \mu \mathrm{M}$ both showed an inhibitive effect on the proliferation of BMSCs in days 7 and 14. The peak of the promotive effect on the proliferation of BMSCs was observed in the group treated with $10 \mu \mathrm{M}$ Morusin. Then, we further investigated the effects of Morusin's concentration (0$50 \mu \mathrm{M})$ on BMSC differentiation and detected the levels of osteogenic marker genes including bone sialoprotein (Bsp), Col1a1, Runx2, and osteocalcin (Ocn). Morusin was found to promote osteogenic marker genes in terms of the expression at $2.5,5$, and $10 \mu \mathrm{M}$, while $10 \mu \mathrm{M}$ of Morusin revealed the strongest effect (Fig. 1c-f). The above results indicated the function of Morusin in promoting the differentiation in addition to proliferation of BMSCs at 2.5-10 $\mu \mathrm{M}$. So, the concentrations of $2.5,5$, and $10 \mu \mathrm{M}$ were selected for subsequent studies.

\section{Morusin promotes osteogenesis of BMSCs in vitro}

Stimulated mRNAs expression of Col1a1, Runx2, and $B s p$ was detected in BMSCs disposed with 2.5, 5, and $10 \mu \mathrm{M}$ of Morusin for 3, 5, and 7 days, in comparison to the control group (Fig. 2a). COL1A1 and RUNX2 protein expression was also found increased in osteogenic BMSCs with Morusin at days 3, 5, and 7 (Fig. 2b). Further, deeper ALP staining was observed in the osteogenic BMSCs after 3 and 10 days of Morusin treatment
(Fig. 2c). Meanwhile, more calcium nodules were also caught sight of in the osteogenic BMSCs disposed with Morusin after 14 days of differentiation, especially in these treated with $10 \mu \mathrm{M}$ Morusin (Fig. 2d). The above results indicated that Morusin could promote the osteogenic differentiation of BMSCs in vitro.

\section{Wnt/ $\beta$-catenin pathway participated in the promoted osteogenic differentiation of BMSCs treated with Morusin in vitro}

Wnt/ $\beta$-catenin signaling pathway is vital for BMSC differentiation and is a significant pathway for promoting new bone formation, inhibiting bone resorption, and maintaining bone mass $[23,24]$. In the current study, increased expression of $\beta$-catenin, lymphoid enhancing factor-1(Lef-1), and dishevelled-1 (Dvl-1) mRNAs were detected in BMSCs treated with Morusin $(0-10 \mu \mathrm{M})$ on the $3 \mathrm{rd}, 5 \mathrm{th}$, and 7 th days of differentiation, respectively (Fig. 3a). Then, further addition of $500 \mathrm{ng} / \mathrm{ml}$ DKK-1, as an effective inhibitor of Wnt / $\beta$-catenin signaling pathway, attenuated such effects of Morusin (10 uM) about the gene expression of $\beta$-catenin, Lef-1, and Dvl-1 (Fig. 3b, c), which was further confirmed by immunofluorescence assay (Fig. 3f). In addition, DKK-1 treatment attenuated ALP staining and calcium nodule deposition in the osteogenic BMSCs treated with Morusin after 3 and 14 days of differentiation, respectively. (Fig. 3d, e). These results suggest the probability of Wnt/ $\beta$-catenin pathway to take part in osteogenic differentiation of promoting effect of Morusin in vitro.

\section{Morusin affected phosphorylation of $\beta$-catenin and nucleus translocation of $\beta$-catenin in vitro}

When Wnt/ $\beta$-catenin signaling pathways are activated, they are often accompanied by phosphorylation inhibition and nuclear transport of $\beta$-catenin. To further explore Morusin's effect on nuclear transport of $\beta$-catenin, we performed immunofluorescence detection of $\beta$-catenin. The consequences revealed that Morusin $(10 \mu \mathrm{M})$ heightened the expression of $\beta$-catenin (red) in the nuclei, while DKK-1 $(500 \mathrm{ng} / \mathrm{ml})$ partially reversed Morusin's effect on $\beta$-catenin translocation (Fig. 4a). Further, it was found that Morusin $(0-10 \mu \mathrm{M})$ had the effect in inhibiting the phosphorylation of $\beta$-catenin. Namely, with the increase of Morusin concentrations, the phosphorylated $\beta$-catenin decreased in terms of the protein expression, while total $\beta$ catenin increased (Fig. 4b). Afterward, the phosphorylation inhibition of $\beta$-catenin by Morusin $(10 \mu \mathrm{M})$ was partially reversed by DKK-1 (500 ng/ml) (Fig. 4c). These results indicated the function of Morusin to activate the Wnt/ $\beta$-catenin signaling pathway via inhibition of $\beta$ catenin phosphorylation and promotion of its nucleus translocation. 


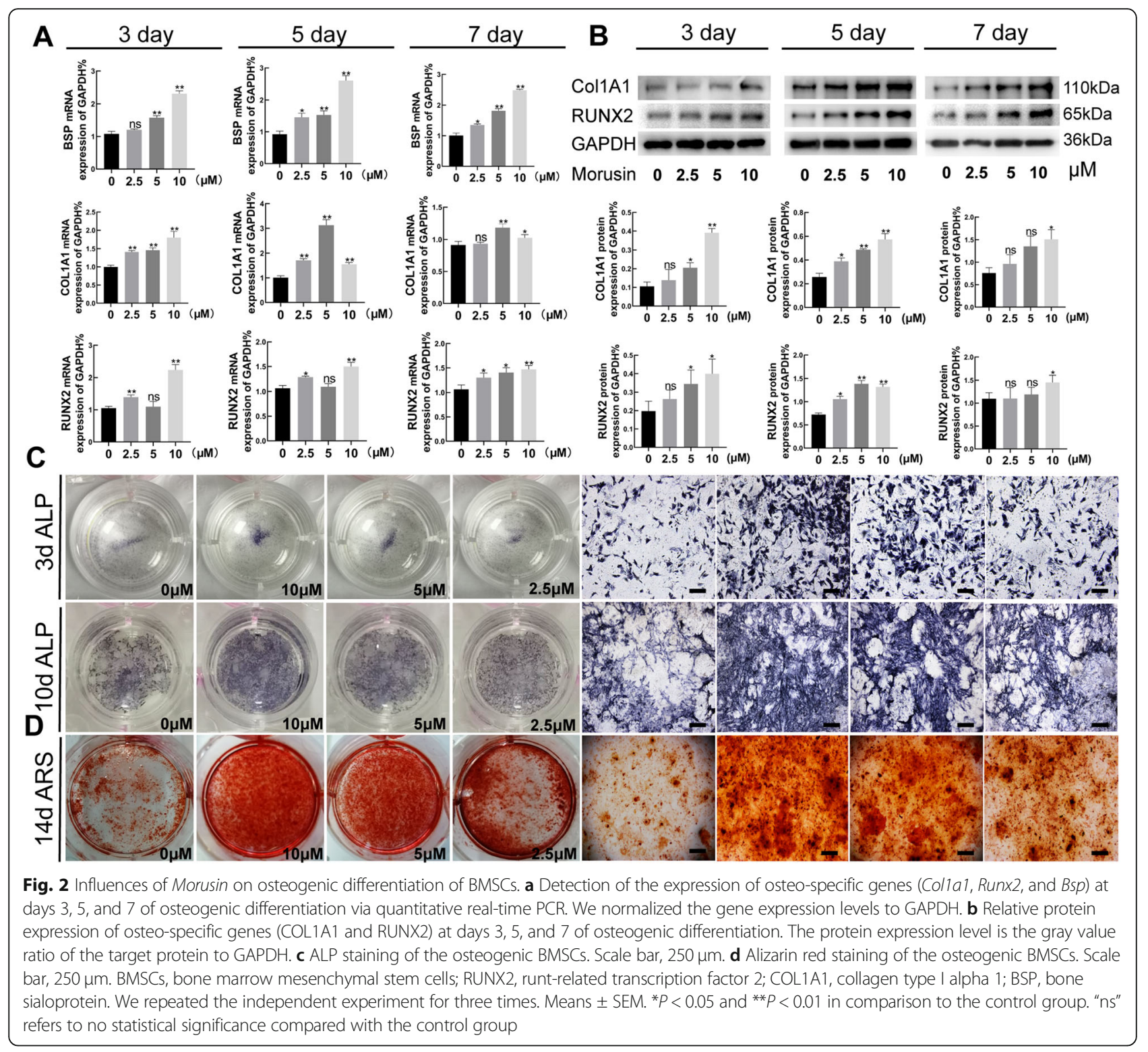

\section{Morusin attenuated bone loss in OVX rats}

Morusin $(40 \mathrm{mg} / \mathrm{kg}$ ) was admitted to the OVX rats to evaluate the effect of Morusin on OP. Micro-CT scan results showed that Morusin ( $40 \mathrm{mg} / \mathrm{kg}$ ) could alleviate the bone loss caused by estrogen deficiency to some extent, which was manifested in increased Tb.N, Tb.Th, BV/TV, and Conn.Dn, while a decrease in Tb.Sp and SMI (Fig. 5a, b). Furthermore, HE staining revealed that in comparison to the OVX group, an increase in the number of bone trabeculae and bone mass indexes (N.Ob/ B.Pm and Ob.S/BS) was observed in the OVX \& Morusin group (Fig. 5c). Similarly, immunohistochemical results also showed that Morusin could attenuate the decrease of the expression of RUNX2 and $\beta$-catenin in the femur of OVX rats (Fig. $5 \mathrm{~d}$ ). Such results indicated that Morusin could attenuate bone loss in OVX rats.
Therefore, we believe that Morusin would be a potential novel candidate for anti-OP.

\section{Discussion}

Due to the high prevalence of osteoporosis in the elderly, the number of patients is still keeping increasing, and over 60 million of osteoporosis patients were reported in China in 2019 [25]. The severe complication of brittle fracture has a higher disability rate and mortality rate, which threatens people's quality of life, as well as their physical and mental health to a great extent [26]. How to prevent and treat osteoporosis effectively has become one of the major clinical and experimental issues. Osteogenesis of osteoblasts and bone resorption of osteoclasts is important for the occurrence and development of osteoporosis [27-29]. As shown in numerous 


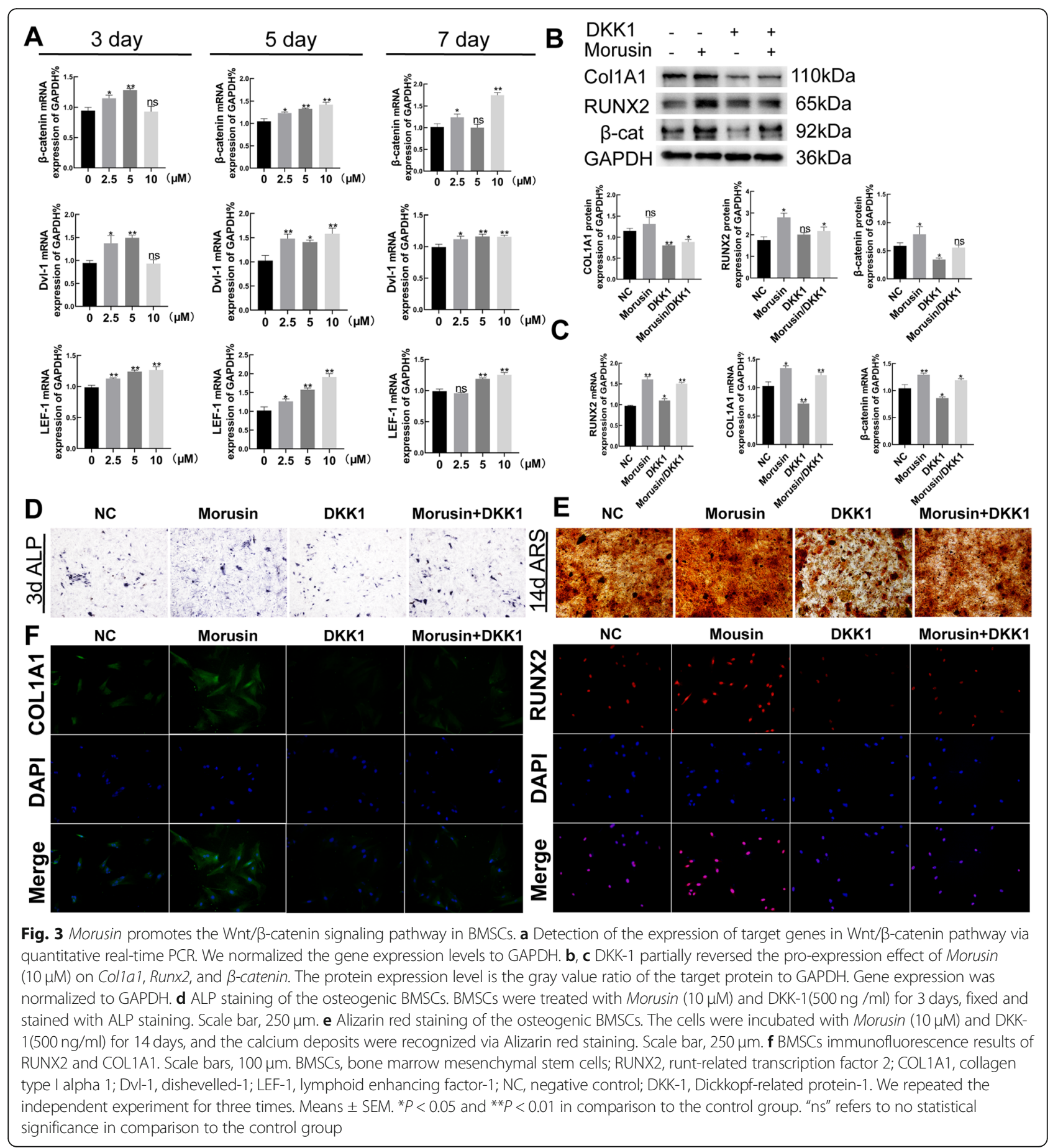

previous studies, promoting BMSC osteogenic differentiation could effectively prevent or slow down the occurrence and development of osteoporosis [30-32]. Morusin, a monomer component from a widely used traditional Chinese medicine Cortex Mori, was proved to be with bioactivities of anti-tumor, anti-inflammatory, and anti-oxidation [33-35]. Although Morusin have been shown to have the function on inhibiting osteoclast differentiation in vitro, there has been no illumination of the function of Morusin about BMSC proliferation and osteogenic differentiation [17]. In this study, how Morusin affect BMSC proliferation was first examined. $10 \mu \mathrm{M}$ Morusin was the optimum concentration to promote BMSC proliferation and had no obvious cytotoxicity on the basis of the results of the MTT assay. Then, we found that Morusin could promote the osteogenic differentiation of BMSCs in the manner of concentration dependence from 2.5 to $10 \mu \mathrm{M}$. Further, we selected 


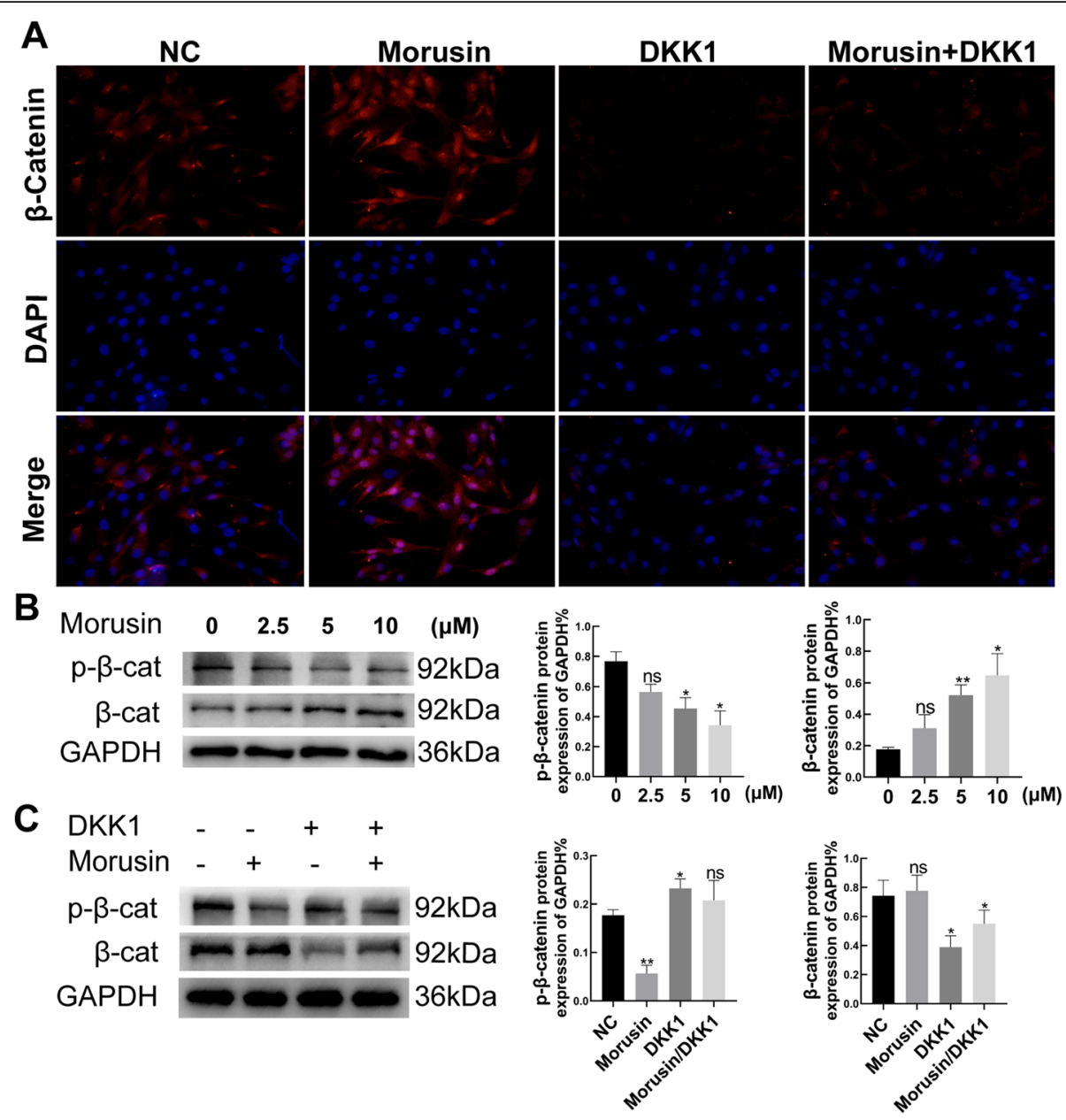

Fig. 4 The effect of Morusin on phosphorylation and nuclear transport of $\beta$-catenin. a Immunofluorescence assay of $\beta$-catenin in the osteogenic BMSCs. Morusin activates nuclear transposition of $\beta$-catenin in BMSCs. Scale bars, $100 \mu \mathrm{m}$. b Total and phosphorylated $\beta$-catenin in the osteogenic BMSCs treated with Morusin $(10 \mu \mathrm{M})$ by Western Blotting. The protein expression level is the gray value ratio of the target protein to GAPDH. $\mathbf{c}$ Total and phosphorylated $\beta$-catenin in the osteogenic BMSCs treated with Morusin $(10 \mu \mathrm{M})$ and DKK-1 $(500 \mathrm{ng} / \mathrm{ml})$ by Western Blotting. The protein expression level is the gray value ratio of the target protein to GAPDH. BMSCs, bone marrow mesenchymal stem cells; NC, negative control; $p$ - $\beta$-catenin, phosphorylated $\beta$-catenin. We repeated the independent experiment for three times. Means \pm SEM. ${ }^{*} P<0.05$ and ${ }^{* *} P<0.01$ in comparison to the control group. "ns" refers to no statistical significance compared with the control group

different differentiation time points of BMSCs to verify the role of Morusin and found that Morusin could promote the expression of osteogenic marker genes at different stages of BMSC differentiation. As an early osteogenic marker, ALP promotes mineralization during osteogenic differentiation. ARS can indicate the number of calcium nodules at the late stage of differentiation [36]. Both of them can directly reflect the effect of osteogenic differentiation. This study showed that Morusin obviously raised the ALP activity and caused the formation of calcium nodules to happen in BMSCs. All the above findings revealed that Morusin was safe and effective in promoting osteogenic differentiation of BMSCs in vitro.

Although Morusin has the ability to effectively advance osteogenic differentiation of BMSCs, its underlying mechanism remains unclear. As confirmed by previous studies, $W n t / \beta$-catenin signaling pathway is a great mediator of BMSCs differentiation into osteoblasts [37-39]. Therefore, we studied the function of Wnt $/ \beta$-catenin signaling pathway in the osteogenesis of BMSCs treated by Morusin. Our results showed that Morusin could significantly increase genes related to $\mathrm{Wnt} / \beta$-catenin signaling in terms of the expression ( $\beta$-catenin, $D v l-1$, and $L E F-1$ ), while DKK-1 can partially block the promoting effect of Morusin on osteogenic differentiation of BMSCs. When $\mathrm{Wnt} / \beta$-catenin signaling pathway is activated, it can lead to the disintegration of APC, Axin, and GSK3 $\beta$ complex. Then, inhibition of $\beta$-catenin phosphorylation raises the levels of $\beta$-catenin in the cell and allows $\beta$-catenin to enter the nuclei to bind to the transcription factor TCF/ LEF, thereby initiating transcription of downstream 


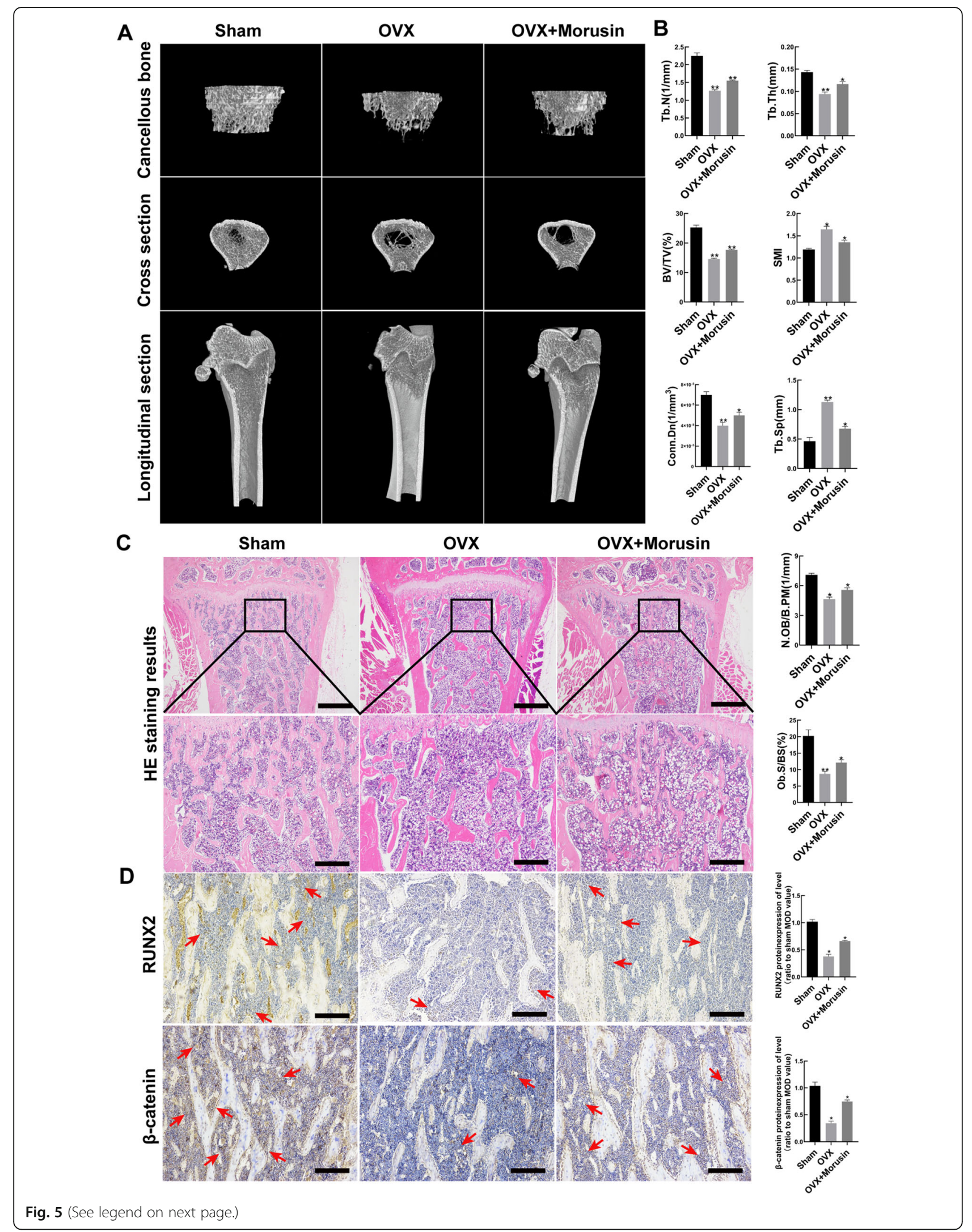


(See figure on previous page.)

Fig. 5 Morusin attenuated bone resorption in OVX rats. a, b Representative three-dimensional reconstruction image and micro-CT analysis of femurs in Sham operated rats, OVX rats, and OVX \& Morusin group. Scale bar, $500 \mu \mathrm{m}$. c Representative images of HE staining of femurs in Sham operated rats, OVX rats and OVX \& Morusin group. Scale bar, $500 \mu \mathrm{m}$. Scale bar (enlarged), $250 \mu \mathrm{m}$. d RUNX2 and $\beta$-catenin immunochemical staining of femurs in Sham operated rats, OVX rats, and OVX \& Morusin group. The red arrows indicate the highly expressed region of RUNX2 and $\beta$-catenin. Scale bar, $100 \mu \mathrm{m}$. OVX, ovariectomized; BV/TV, bone volume/tissue volume; Tb.N, trabecular number; Tb.Th, trabecular thickness; Tb.Sp, trabecular separation; Conn.Dn, connectivity density; SMI, structure model index; RUNX2, runt-related transcription factor 2; N.Ob/B.Pm, osteoblasts number/bone perimeter; Ob.S/BS, osteoblast surfaces/bone surface. We repeated the independent experiment for three times. Means \pm SEM. ${ }^{*} P<0.05$ and ${ }^{* *} P<0.01$ in comparison to the control group

target genes [40]. Thus, we further examined phosphorylation levels of $\beta$-catenin and its nucleus translocation. As per these results, Morusin had an impact of inhibition about the phosphorylation of $\beta$-catenin but promoted its nucleus translocation. These findings revealed that Morusin had the ability in advancing osteogenic differentiation of BMSCs via activating $\mathrm{Wnt} / \beta$-catenin signaling pathway.

The impact of Morusin on bone loss in rats was further investigated. In this research, OVX rats were used as the osteoporosis model, which has been widely regarded as a model with low osteogenic capacity of the BMSCs [37, 41]. Micro-CT, HE staining, and immunohistochemical analysis showed that after Morusin treatment, the bone loss of OVX rats was reduced, the number of osteoblasts was increased, and the protein expression of osteogenic marker genes was also increased. These evidences show that Morusin can promote osteoblastic proliferation and bone formation in OVX rats, which is consistent with vitro experiment.

\section{Conclusion}

It could be concluded that our current study demonstrated the ability of Morusin in promoting the osteogenic differentiation in addition to proliferation of BMSCs via activation of $\mathrm{Wnt} / \beta$-catenin signaling pathway in vitro, and also presented anti-osteoporosis activity in OVX rats. This study also suggested the potential of Morusin to be a therapeutic agent for osteoporosis. Further researches are needed before its clinical application.

\footnotetext{
Abbreviations

BMSCs: Bone marrow mesenchymal stem cells; OP: Osteoporosis; DKK1: Dickkopf-related protein-1; LRP5: Lipoprotein receptor-associated protein 5; DMSO: Dimethylsulfoxide; OGM: Osteogenic growth medium; ARS: Alizarin red staining; RT-qPCR: Quantitative real-time PCR; GAPDH: Glyceraldehyde phosphate dehydrogenase; COL1A1: Collagen type I alpha 1; RUNX2: Runtrelated transcription factor 2; BSP: Bone sialoprotein; OCN: Osteocalcin; Dvl1: Dishevelled-1; LEF-1: Lymphoid enhancing factor-1; OVX: Ovariectomized; N.Ob/B.Pm: Osteoblasts number/bone perimeter; Ob.S/BS: Osteoblast surfaces/bone surface; Tb.Sp: Trabecular separation; Tb.N: Trabecular number; BV/TV: Bone volume/tissue volume; Conn.Dens: Connectivity density; Tb.Th: Trabecular thickness; SMI: Structure model index
}

\section{Authors' contributions}

Chen L.B., Wen Y.X., and Chen M. designed the research study; Chen M. and Han H. performed the research; Chen M., Han H., and Zhou S.Q. analyzed the data; Chen M., Han H., Chen L.B., and Wen Y.X. wrote and revised the paper. All authors read and approved the final manuscript.

\section{Funding}

Thanks for the support given to this work by the National Natural Science Foundation of China (No. 81673490, 81603214, 81972036), the Key Research and Development Project of Hubei province (No. 2020BCA071), and the National Key Research and Development Project of China (No.

2020YFA0803900)

\section{Availability of data and materials}

The datasets used and/or analyzed during the current study are available from the corresponding author on reasonable request.

\section{Declarations}

\section{Ethics approval and consent to participate}

All animal experiments were conducted in the Center for Animal Experiment of Wuhan University (Wuhan, China), and all protocols were authorized by the Animal Welfare Committee in Wuhan University (License number: 14016).

\section{Consent for publication}

Not applicable.

\section{Competing interests}

The authors declare that they have no competing interests.

\section{Author details}

${ }^{1}$ Department of Joint Surgery and Sports medicine, Zhongnan Hospital of Wuhan University, Wuhan 430071, China. ${ }^{2}$ Hubei Provincial Key Laboratory of Developmentally Originated Disease, Wuhan 430071, China. ${ }^{3}$ Department of Orthopedics Department, Renmin Hospital of Wuhan University, Wuhan 430060, China.

Received: 22 January 2021 Accepted: 24 February 2021

Published online: 12 March 2021

\section{References}

1. Cummings SR, Melton LJ. Epidemiology and outcomes of osteoporotic fractures. Lancet. 2002;359(9319):1761-7.

2. Zhang X, Xu H, Li GHY, Long MT, Cheung CL, Vasan RS, et al. Metabolomics insights into osteoporosis through association with bone mineral density. J Bone Miner Res. 2021.

3. Rachner TD, Khosla S, Hofbauer LC. Osteoporosis: now and the future. Lancet. 2011;377(9773):1276-87.

4. Zhang LK, Chen WY, Wang HM, Liu C, He J, Tang Y, et al. Growth factors regional patterned and photoimmobilized scaffold applied to bone tissue regeneration. J Mater Chem B. 2020;8(48):10990-1000.

5. Cai H, Zou J, Wang W, Yang A. BMP2 induces hMSC osteogenesis and matrix remodeling. Mol Med Rep. 2021; 23(2).

6. Sharma AK, Roberts RL, Benson RD Jr, Pierce JL, Yu K, Hamrick MW, et al. The senolytic drug navitoclax (ABT-263) causes trabecular bone loss and impaired osteoprogenitor function in aged mice. Front Cell Dev Biol. 2020;8: $35-40$. 
7. Lin Z, He H, Wang M, Liang J. MicroRNA-130a controls bone marrow mesenchymal stem cell differentiation towards the osteoblastic and adipogenic fate. Cell Prolif. 2019;52(6):126-88.

8. Canalis E. Wnt signalling in osteoporosis: mechanisms and novel therapeutic approaches. Nat Rev Endocrinol. 2013;9(10):575-83.

9. Oichi T, Otsuru S, Usami Y, Enomoto-Iwamoto M, Iwamoto M. Wnt signaling in chondroprogenitors during long bone development and growth. Bone. 2020;137:1153-68

10. Huybrechts Y, Mortier G, Boudin E, Van Hul W. WNT signaling and bone: lessons from skeletal dysplasias and disorders. Front Endocrinol. 2020;11: $165-8$.

11. Zhao C, Yu T, Dou Q, Guo Y, Yang X, Chen Y. Knockout of TLR4 promotes fracture healing by activating Wnt/ $\beta$-catenin signaling pathway. Pathol Res Pract. 2020;216(2):1527-66.

12. Shi $L$, Feng $L$, Zhu ML, Yang ZM, Wu TY, Xu J, et al. Vasoactive intestinal peptide stimulates bone marrow-mesenchymal stem cells osteogenesis differentiation by activating Wnt/ $\beta$-catenin signaling pathway and promotes rat skull defect repair. Stem Cells Dev. 2020;29(10):655-66

13. Choi DW, Cho SW, Lee SG, Choi CY. The beneficial effects of Morusin, an isoprene flavonoid isolated from the root bark of Morus. Int J Mol Sci. 2020; 21(18):431-42

14. Yang C, Luo J, Luo X, Jia W, Fang Z, Yi S, et al. Morusin exerts anti-cancer activity in renal cell carcinoma by disturbing MAPK signaling pathways. Ann Transl Med. 2020;8(6):32-7.

15. Chen C, Wang J, Chen J, Zhou L, Wang H, Chen J, et al. Morusin alleviates mycoplasma pneumonia via the inhibition of Wnt/ $\beta$-catenin and NF-KB signaling. Biosci Rep. 2019;39(6):657-73.

16. Zhang Y, Weng Q, Chen J, Han J. Morusin inhibited human osteosarcoma via PI3K-AKT signaling pathway. Curr Pharm Biotechnol. 2020;9(6):52-7.

17. Lin B, Huang JF, Liu XW, Ma XT, Liu XL, Lu Y, et al. Rapid, microwaveaccelerated synthesis and anti-osteoporosis activities evaluation of Morusin scaffolds and Morusignin L scaffolds. Bioorg Med Chem Lett. 2017;27(11): 2389-96.

18. Bao J, Ma C, Ran J, Xiong Y, Yan S, Wu L. Wnt/B-catenin and hedgehog pathways are involved in the inflammatory effect of interleukin 18 on rat chondrocytes. Oncotarget. 2017;8(66):109962-72.

19. Zhou DA, Zheng HX, Wang CW, Shi D, Li JJ. Influence of glucocorticoids on the osteogenic differentiation of rat bone marrow-derived mesenchymal stem cells. BMC Musculoskelet Disord. 2014;15:23-9.

20. Hang K, Ye C, Xu J, Chen E, Wang C, Zhang W, et al. Apelin enhances the osteogenic differentiation of human bone marrow mesenchymal stem cells partly through Wnt/ $\beta$-catenin signaling pathway. Stem Cell Res Ther. 2019; 10(1):18-9.

21. Jia $Y$, He W, Zhang H, He L, Wang Y, Zhang T, et al. Morusin ameliorates IL$1 \beta$-induced chondrocyte inflammation and osteoarthritis via NF-KB signal pathway. Drug Des Devel Ther. 2020;14:1227-40.

22. Dempster DW, Compston JE, Drezner MK, Glorieux FH, Kanis JA, Malluche $H$, et al. Standardized nomenclature, symbols, and units for bone histomorphometry: a 2012 update of the report of the ASBMR Histomorphometry Nomenclature Committee. J Bone Miner Res. 2013;28(1):2-17.

23. Shen G, Ren H, Shang Q, Zhao W, Zhang Z, Yu X, et al. Foxf1 knockdown promotes BMSC osteogenesis in part by activating the Wnt/ $\beta$-catenin signalling pathway and prevents ovariectomy-induced bone loss. EBioMedicine. 2020;52:10262-6

24. Sun W, Shi Y, Lee WC, Lee SY, Long F. Rictor is required for optimal bone accrual in response to anti-sclerostin therapy in the mouse. Bone. 2016;85:1-8.

25. Zeng Q, Li N, Wang Q, Feng J, Sun D, Zhang Q, et al. The prevalence of osteoporosis in China, a nationwide, multicenter DXA survey. J Bone Miner Res. 2019:34(10):1789-97.

26. Seeman E, Martin TJ. Antiresorptive and anabolic agents in the prevention and reversal of bone fragility. Nat Rev Rheumatol. 2019;15(4):225-36.

27. Armas $L A$, Recker RR. Pathophysiology of osteoporosis: new mechanistic insights. Endocrinol Metab Clin N Am. 2012;41(3):475-86.

28. Teitelbaum SL. Bone resorption by osteoclasts. Science. 2000;289(5484): 1504-8.

29. Ducy P, Schinke T, Karsenty G. The osteoblast: a sophisticated fibroblast under central surveillance. Science. 2000;289(5484):1501-4.

30. Zhang J, Zhang W, Dai J, Wang X, Shen SG. Overexpression of Dlx2 enhances osteogenic differentiation of BMSCs and MC3T3-E1 cells via direct upregulation of Osteocalcin and Alp. Int J Oral Sci. 2019;11(2):12-7.
31. Li LY, Wang XL, Wang GS, Zhao HY. MiR-373 promotes the osteogenic differentiation of BMSCs from the estrogen deficiency induced osteoporosis. Eur Rev Med Pharmacol Sci. 2019;23(17):7247-55.

32. Zhou J, Nie H, Liu P, Wang Z, Yao B, Yang L. Down-regulation of miR-339 promotes differentiation of BMSCs and alleviates osteoporosis by targeting DLX5. Eur Rev Med Pharmacol Sci. 2019;23(1):29-36.

33. Park HJ, Min TR, Chi GY, Choi YH, Park SH. Induction of apoptosis by morusin in human non-small cell lung cancer cells by suppression of EGFR/ STAT3 activation. Biochem Biophys Res Commun. 2018;505(1):194-200.

34. Cheng PS, Hu CC, Wang CJ, Lee YJ, Chung WC, Tseng TH. Involvement of the antioxidative property of morusin in blocking phorbol ester-induced malignant transformation of JB6 $\mathrm{P}(+)$ mouse epidermal cells. Chem Biol Interact. 2017:264:34-42.

35. Lim SL, Park SY, Kang S, Park D, Kim SH, Um JY, et al. Morusin induces cell death through inactivating STAT3 signaling in prostate cancer cells. Am J Cancer Res. 2015;5(1):289-99.

36. Jo S, Kang S, Han J, Choi SH, Park YS, Sung IH, et al. Accelerated osteogenic differentiation of human bone-derived cells in ankylosing spondylitis. J Bone Miner Metab. 2018;36(3):307-13.

37. Wang Y, Zhang $X$, Shao J, Liu H, Liu X, Luo E. Adiponectin regulates BMSC osteogenic differentiation and osteogenesis through the Wnt/ $\mathrm{B}$-catenin pathway. Sci Rep. 2017;7(1):36-52.

38. Liang T, Gao W, Zhu L, Ren J, Yao H, Wang K, et al. TIMP-1 inhibits proliferation and osteogenic differentiation of hBMSCs through Wnt/ $\beta$ catenin signaling. Biosci Rep. 2019;39(1):48-59.

39. Zhou P, Li Y, Di R, Yang Y, Meng S, Song F, et al. H19 and Foxc2 synergistically promotes osteogenic differentiation of BMSCs via Wnt- $\beta$ catenin pathway. J Cell Physiol. 2019;234(8):13799-806.

40. Ahmadzadeh A, Norozi F, Shahrabi S, Shahjahani M, Saki N. Wnt/ $\beta$-catenin signaling in bone marrow niche. Cell Tissue Res. 2016;363(2):321-35.

41. Qi M, Zhang L, Ma Y, Shuai Y, Li L, Luo K, et al. Autophagy maintains the function of bone marrow mesenchymal stem cells to prevent estrogen deficiency-induced osteoporosis. Theranostics. 2017:7(18):4498-516.

\section{Publisher's Note}

Springer Nature remains neutral with regard to jurisdictional claims in published maps and institutional affiliations.
Ready to submit your research? Choose BMC and benefit from:

- fast, convenient online submission

- thorough peer review by experienced researchers in your field

- rapid publication on acceptance

- support for research data, including large and complex data types

- gold Open Access which fosters wider collaboration and increased citations

- maximum visibility for your research: over $100 \mathrm{M}$ website views per year

At $\mathrm{BMC}$, research is always in progress.

Learn more biomedcentral.com/submissions 